\title{
ESTIMACIÓN DE PARÁMETROS GENÉTICOS PARA PESO AL DESTETE EN GANADO BLANCO OREJINEGRO (BON) EN EL NOROCCIDENTE COLOMBIANO
}

\author{
ESTI MATI ON OF GENETIC PARAMETERS FOR WEANI NG \\ WEIGHT I N WHITE OREJ I NEGRO CATTLE I N THE COLOMBIAN \\ NORTHWEST
}

Jhon Cañas Á,*1 Zoot, Julian Ramirez T, ${ }^{1}$ Zoot, Oscar Arboleda A, ${ }^{2}$ Zoot, Jorge Ochoa S, ${ }^{2}$ Zoot, Oscar Vergara G, ${ }^{1,3}$ Esp, Mario Cerón-Muñoz ${ }^{1,2}$. Ph.D.

\begin{abstract}
${ }^{1}$ Universidad de Antioquia, Facultad de Ciencias Agrarias e Instituto de Biología, Grupo de investigación en Genética y Mejoramiento Animal. Medellín, Colombia. ${ }^{2}$ Universidad de Antioquia, Facultad de Ciencias Agrarias. Medellín, Colombia. ${ }^{3}$ Universidad de Córdoba, Facultad de Medicina Veterinaria y Zootecnia. Departamento de Ciencias Pecuarias. Montería, Colombia.*Correspondencia: jhonjacobo@gmail.com
\end{abstract}

Recibido: Septiembre 20 de 2007; Aceptado: Enero 10 de 2008

\section{RESUMEN}

Objetivo. Estimar los componentes de varianza genético aditivo directo y materno para peso al destete en ganado criollo Blanco Orejinegro (BON). Materiales y métodos. Se utilizaron 356 registros entre los años 1988 al 2007, del núcleo de animales BON puro de la hacienda Vegas de la Clara de la Universidad de Antioquia. El análisis fue realizado utilizando un modelo animal incluyendo los efectos fijos de sexo y época de destete, y como covariable la edad al destete y los efectos aleatorios genético directo, genético materno, ambiente permanente y residual. Resultados. El peso promedio al destete fue de $196.3 \pm 31.4 \mathrm{~kg}$, a una edad promedio de $271.8 \pm 13.5$ días. Se encontraron 21 animales endogámicos con un coeficiente de endogamia de $24.5 \%$. La heredabilidad directa y materna fueron de $0.63 \pm 0.36$ y $0.22 \pm 0.19$ con una correlación entre el efecto directo y el materno de $-0.78 \pm 0.21$. Conclusión. De acuerdo a los resultados, existe variabilidad genética en el núcleo BON para esta característica.

Palabras clave: Efecto materno, endogamia, genética, ganado de carne, heredabilidad. 


\section{ABSTRACT}

Objective. To estimate the direct and maternal additive genetic components of variance for weaning weight in native cattle $e_{\llcorner}$Blanco Orejinegro (BON). Materials and methods. 356 records of the animal nucleus pure breed BON belonging to Vegas de la Clara farm, property of Universidad de Antioquia, between the years 1988 to 2007 were used. Analysis was made using an animal model including sex and weaning time fixed effects, and as covariable weaning age and as random effects, direct genetic, maternal genetic, permanent environmental and residual. Results. Mean weaning weight was $196.3 \pm 31.4 \mathrm{~kg}$ at a mean age of $271.8 \pm 13.5$ days. 21 inbreeding animals had an inbreeding coefficient of $24.5 \%$. Direct and maternal heritability was of $0.63 \pm 0.36$ and $0.22 \pm 0.19$ respectively, with a correlation between the direct and maternal effect of $-0.78 \pm 0.21$. Conclusions. According to these results, genetic variability exists in the nucleus BON for this characteristic.

Key words: Maternal effect, inbreeding, genetic, beef cattle, heritability.

\section{NTRODUCCIÓN}

El potencial genético de las razas criollas colombianas radica en tener más de 500 años de adaptación a las condiciones agroecológicas nacionales, tiene alta longevidad, resistencia a algunas enfermedades, baja mortalidad y alta eficiencia productiva (1-3). Asimismo, las razas criollas colombianas tienen una gran ventaja competitiva para conquistar el mercado de la Unión Europea, gracias a la calidad de su carne, que está a la altura de las mejores del mundo (4).

Son escasos los datos que hacen referencia al potencial genético de las razas criollas y que además sirvan de base para futuros programas de mejora genética. En especial, en características tan importantes para la selección de bovinos de carne como el peso de los terneros al destete, componente básico del desempeño productivo que inciden en el retorno neto de la explotación (5).

El peso al destete es una medida que permite evaluar la habilidad materna (efecto genético aditivo materno) y la capacidad de desarrollo propia del ternero aportado por la mitad del valor genético aditivo del padre y la mitad del valor genético aditivo de la madre (efecto genético aditivo directo) $(2,6)$. Además es una característica empleada en la mayoría de ganaderías de cría para realizar la preselección de sus futuros reproductores; ya que al destete el animal ha alcanzado alrededor del $50 \%$ de su peso final.

En la práctica, las evaluaciones genéticas que emplean la característica peso al destete pocas veces incluyen el efecto materno, el cual en conjunto con el efecto genético directo podrían dar una respuesta económica mayor, debido al progreso genético que es alcanzado al momento de la selección $(6,7)$. La no inclusión de los efectos genéticos maternos y del ambiente permanente puede llegar a sobreestimar el componente del efecto genético directo (6).

El objetivo de este estudio fue estimar los componentes de varianza genético aditivo directo y materno para peso al destete en ganado criollo Blanco Orejinegro (BON).

\section{MATERI ALES Y MÉTODOS}

Sitio de Estudio. Se utilizaron 356 registros entre los años 1988 al 2007, provenientes del núcleo de animales BON puro de la hacienda Vegas de la Clara de la Universidad de Antioquia. La hacienda se encuentra localizada en el valle del rio Porce, nordeste antioqueño, con una temperatura media de 
27ํㅡ, precipitación de 2000 mm/año, altitud de 1000 msnm, humedad relativa del $80 \%$ y clasificación agro-ecológica de Bosque Húmedo Premontano Bajo (bh-PMB).

Animales. Los animales pastorean bajo un sistema rotacional en pasturas de Brachiaria decumbens (pasto amargo), Brachiaria humidícola ( pasto dulce), Brachiaria arrecta (urare), Cynodon nlemfluensis (pasto estrella) y Paspalum sp (gramas nativas).

Análisis estadistico. Se utilizó un modelo animal incluyendo los efectos fijos de sexo y época de destete, como covariable la edad al destete y los efectos aleatorios genético aditivo directo, genético aditivo materno, ambiente permanente y residual. Para este estudio no se consideró el efecto número de partos o edad madre, por no disponer de esta información en la base de datos. El análisis fue realizado con el programa MTDFREML desarrollado por Boldman et al (8) con el cual se obtuvieron los componentes de (co)varianza y los valores de cría; estos últimos se dividieron entre dos para calcular los DEP's. El modelo para peso al destete en notación matricial fue el siguiente:

$$
y=X \beta+b(X-\bar{X})+Z a+W m+S p e+e
$$

donde:

y = vector de observaciones (peso al
destete); $\beta=$ vector de efectos fijos;

$\mathrm{b}(\mathrm{X}-\bar{\chi})=$ coeficiente de regresión parcial de la edad al destete, siendo $X$ los días de edad de destete y $\bar{\chi}$ el promedio de los días de edad al destete;

$a=$ vector de efectos aleatorios genéticos aditivos directos.

$m=$ vector de efectos aleatorios genético aditivo materno.

pe $=$ vector de efectos de ambiente permanente.

$\mathrm{e}=$ vector de efectos aleatorios residuales

$\mathrm{X}, \mathrm{Z}, \mathrm{W}, \mathrm{S}=$ son matrices de incidencia que relacionan los efectos fijos, genético aditivo directo, genético aditivo materno y de ambiente permanente con el vector de observaciones.

\section{RESULTADOS}

Se encontraron en la matriz de parentesco seis generaciones en el pedigrí, donde 21 animales presentaron un coeficiente de endogamia de $24.5 \%$. El peso promedio al destete fue de $196.3 \pm 31.4 \mathrm{~kg}$ a una edad promedio de $271.8 \pm 13.5$ días.

El efecto sexo mostró diferencias significativas a favor de los machos $(P \leq 0,05)$, correspondiendo a $15.1 \mathrm{~kg}$ (Tabla 1). Caso contrario ocurrió con el efecto época de destete, ya que no se encontró diferencias significativas entre la época seca y la de lluvias.

Tabla 1. Número de observaciones ( $n$ ) y media $(m)$ estimados para los efectos fijos sexo y época de destete en la variable peso al destete.

\begin{tabular}{cccc}
\hline \multicolumn{2}{c}{ Efectos fijos } & $\mathbf{n}$ & $\boldsymbol{\mu}(\mathbf{K g})$ \\
\hline \multirow{2}{*}{ Sexo } & Machos & 95 & $204.96^{\circ}$ \\
& Hembras & 129 & $189.9^{\circ}$ \\
\hline \multirow{2}{*}{ Época } & Luvias & 143 & $199.95^{\circ}$ \\
& Seca & 81 & $189.81^{\circ}$ \\
\hline
\end{tabular}

Medias con letras diferentes en la misma columna difieren significativamente $(p \leq 0,05)$.

La heredabilidad directa para el peso al destete fue de $0.63 \pm 0.36$ (media-alta) y la materna de $0.22 \pm 0.19$ (baja) con una correlación entre ambas de -0.78 \pm 0.21 .

Se calcularon las diferencias esperadas predichas (DEP's) directas y maternas y sus confiabilidades para el peso al destete en todos los animales. El promedio de las DESP's directas fue de $2.95 \pm 6.72$ y el de los maternos fue de $-1.02 \pm 3.47$. En la figura 1 se muestra la correlación negativa entre las DEP's directas y maternas.

Se presentó una relación positiva entre DEP's directa y la generación, mostrando que en el transcurso de las seis generaciones evaluadas se ha venido obteniendo mejores pesos al destete (Figura 2). Por el contrario, 


\section{DEP'S Directas vs DEP'Maternas}

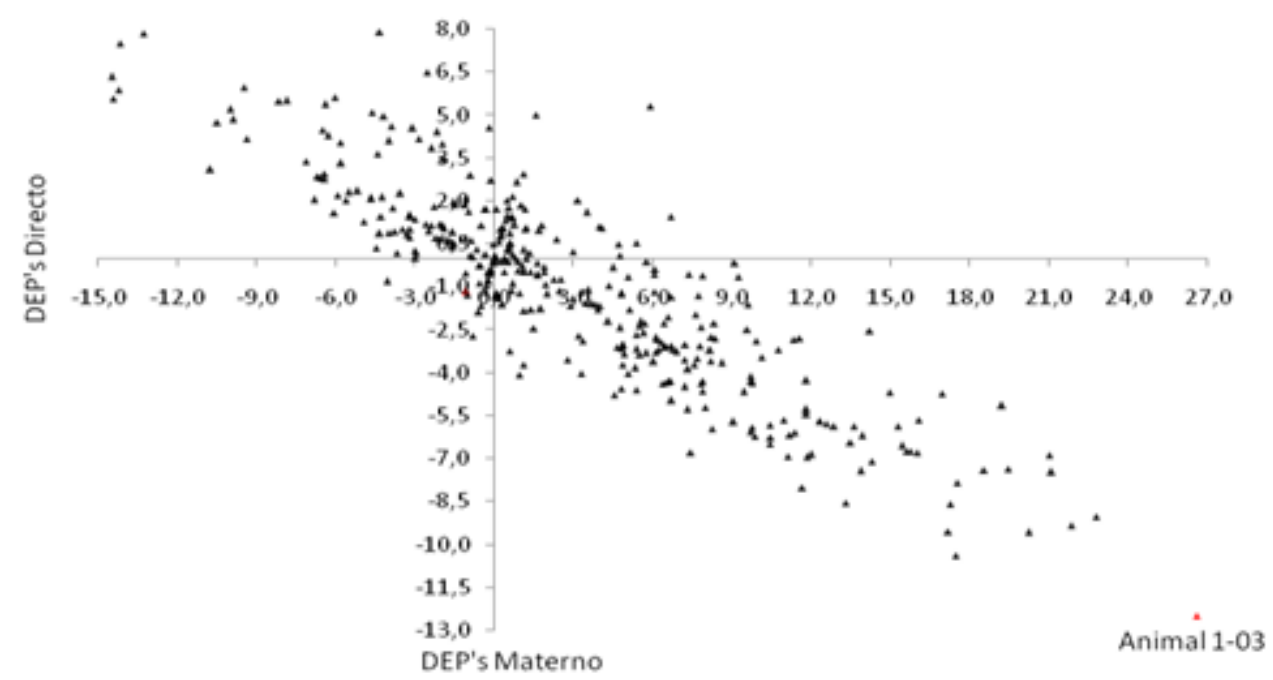

Figura 1. Relación entre DEP's directa y DEP's materna hallados en la población.
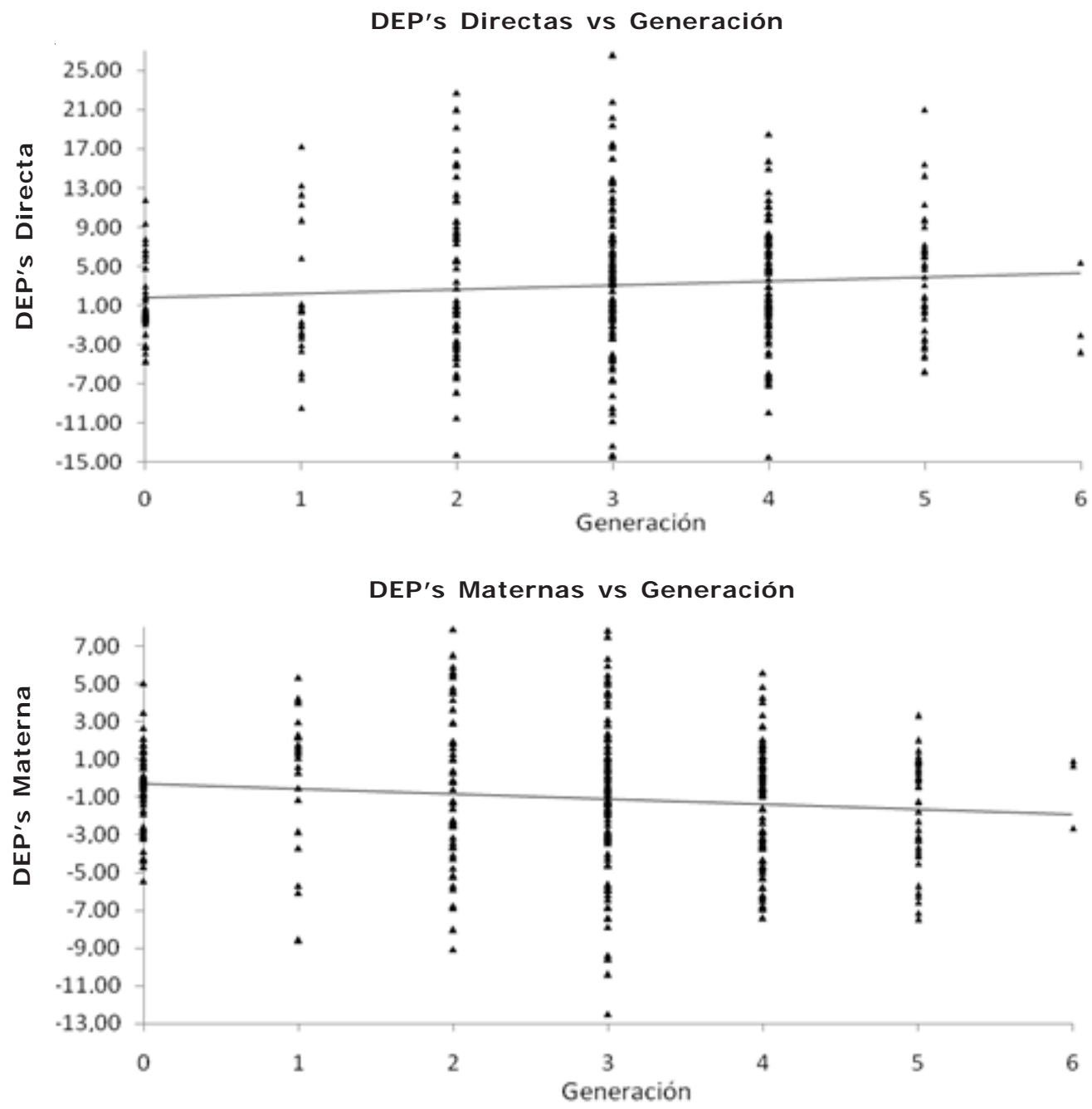

Figura 2. Correspondencia entre DEP's directas y maternas con relación a la generación. 
se presentó una relación negativa entre DEP's materno y la generación.

\section{DISCUSIÓN}

El porcentaje de consanguinidad encontrado es alto, ya que su población actual es muy reducida, lo que ha conllevado a altos grados de consanguinidad (3).

El promedio del peso al destete se encuentra dentro del rango de valores para esta característica reportados para otras razas criollas de I beroamérica. Botero (9), reportó en BON pesos al destete entre 179 y 200 kg. Ossa y Perez (10) en ganado Costeño con Cuernos encontraron pesos al destete de $173.3 \pm 23 \mathrm{~kg}$ ajustados a 240 días. Otros autores (11-16) reportaron promedios que variaron entre $155 \mathrm{~kg}$ y $210 \mathrm{~kg}$. Cienfuegos et al (5) reportaron un peso al destete menor al de este estudio $(210.5 \pm 41.4 \mathrm{~kg}$. ajustado a los 205 días de edad), en las razas Angus, Hereford y Polled Hereford. En animales cruzados se resaltan los altos pesos logrados en cruces de razas criollas con Cebú, donde se alcanzan pesos hasta de $214 \mathrm{~kg}$ al destete (17).

La diferencia encontrada en el peso al destete entre machos y hembras, es debido al mayor potencial de los machos para ganar peso con referencia a las hembras debido al dimorfismo sexual. Estos resultados coinciden con la mayoría de las publicaciones que consideran el efecto sexo sobre el peso al destete. Hernández (2) en Romosinuano, Martínez et al (18) en la raza BON, Kaps et al (19) en Angus y Ossa et al (15), en Romosinuano, reportaron diferencias entre machos y hembras que oscilan entre $15 \mathrm{y}$ $50 \mathrm{~kg}$., a favor de los machos.

Con respecto a la no significancia de la época sobre el peso al destete, se debió a la disponibilidad de forraje para los animales en las dos épocas. A diferencia de este estudio, se han encontrado efectos significativos de la época de destete sobre el peso al destete en ganado Romosinuano, Nelore y Mestizo, $(14,20,21)$.
Se han reportado valores de heredabilidad directa y materna similares, inferiores y superiores a los encontrados en este estudio, como es el caso de Splan et al (22) quienes estimaron en ganado cruzado heredabilidades directas y maternas para peso al destete de $0.4 \pm 0.02$ y $0.19 \pm 0.02$ respectivamente, con una correlación entre ambas de $-0.18 \pm 0.08$. Valores inferiores para la heredabilidad directa y materna fueron reportados por Quintero et al (6) al obtener coeficientes de heredabilidad directa de 0.10 a 0.29 y de heredabilidad materna de 0.07 a 0.17 , con correlaciones de -0.18 a -0.29, en diferentes modelos estadísticos empleados en ganado Brahman y Cebú comercial. Asimismo, Elzo y Wakeman (23) en Angus y Brahman encontraron heredabilidades directas de 0.25 y 0.29 y maternas de 0.17 y 0.18 , respectivamente; y Ossa et al (15) con valores de heredabilidad directa de $0.14 \pm 0.05$ y materna de $0.12 \pm 0.03$ en ganado Romosinuano.

Como la heredabilidad es un parámetro que varía mucho entre poblaciones y su obtención se realiza con el único fin de determinar los métodos de selección de una población en particular, se encontraron otros valores de heredabilidad directa inferiores a los aquí enunciados y que variaron desde 0.08 hasta 0.59 reportados por diferentes autores tanto en razas criollas como foráneas: desde 0.16 a 0.38 en Nellore (24); en San Martinero y Cebú con 0.10 y 0.08 , respectivamente (25); en Angus una heredabilidad directa de 0.21 y 0.23 en Polled Hereford (5). Por otro lado, se han reportaron altas heredabilidades del peso al destete ajustado a los 205 días en Angus con rangos de 0.53 a 0.59 (19).

Es importante la estimación tanto de heredabilidades directas como maternas, ya que es bien sabido que el desarrollo del ternero no solo es afectado por el genotipo propio del animal heredado de sus padres (efecto directo), sino también del medio ofrecido por su madre (efecto materno). La correlación negativa entre ambas características indica que los reproductores que mejoran pesos al destete en su descendencia no serán buenos en habilidad materna. Estos efectos antagonistas entre 
efectos directos y maternos han sido reportados por otros autores $(26,27)$.

El promedio de DEP's directas es similar al reportado en la raza Hereford (28) y Limousine (29), pero inferior al reportado en las razas Angus y Tarentaise (29) y superior al encontrado en las razas Simental (28) y Gelbvieh (29), indicando la gran variabilidad que existe para las DEP's del peso al destete entre razas y poblaciones. En la figura 1 se observa que a medida que aumenta las DEP's directas disminuyen las DEP's maternas. Esto se debe a que son características completamente opuestas, ya que un animal que le aporta buenos pesos al destete a sus crías, será malo para dar crías hembras con buena habilidad materna. Tomando como ejemplo el animal 1-03 con la mejor DEP's directa (Figura 1), este aporta a todos sus hijos (machos y hembras) $26.57 \mathrm{~kg}$ más que el promedio actual de peso al destete de la hacienda con una confiabilidad del 0.71 , pero sus crías hembras serán malas en habilidad materna y darán crías 12.49 kg menos pesadas que el promedio.

La elección de la herramienta por la cual se realice la selección de los futuros reproductores (DEP's directas o maternas) dependerá del productor y de los objetivos que tenga planteados para su hato. Por lo tanto, para la selección de reproductores se deben considerar las DEP's directas y para la selección de hembras de remplazo las DEP's maternas, aprovechando para ello la variabilidad que presentan éstos en la población, especialmente las DEP's directas.

Con respecto a la relación entre DEP's directas y maternas y las diferentes generaciones de la población, se evidencia que en el transcurrir de las generaciones, se ha venido presentando en el hato una disminución en la habilidad materna (Figura 2).

En conclusión, mediante el uso de la evaluación genética se podrá tener mejores criterios de selección de los animales como futuros reproductores, además, permitirá obtener una mejor venta de terneros destetados por su valor genético. Es posible la realización de mejoramiento por selección por medio de las DEP's directas para peso al destete, debido a la alta heredabilidad directa (0.63), la cual es considerada alta en comparación con otros estudios. Con lo anterior se espera un progreso genético alto para los próximos años en la Hacienda Vegas de la Clara.

\section{REFERENCI AS}

1 Arboleda O. Correlaciones entre pesos vivos y medidas corporales del ganado Blanco-Orejinegro a diferentes edades. Rev Fac Nal Agr Medellín 1979; XXXII (2): 27-39.

2 Hernández BG. Genetic factors in beef cattle crosses in Colombia. Dissertation. Colorado State University. Colorado. USA: Fort Collins; 1976.

3 López A, Saldarriaga O, Arango A, Rugeles $M$, Zuluaga $F$, Olivera $M$, Bermúdez N, Bedoya G, Ossa J. Ganado Blanco Orejinegro (BON): Una alternativa para la producción en Colombia. Rev Col Cienc Pec 2001; 14(2).
4 Noticias SNE. Presidencia de la República de Colombia, Agosto 3 del 2005. [Fecha de Acceso, 11 de septiembre del 2007]. URL disponible en: http:// www.presidencia.gov.co/prensa_new/ sne/2005/agosto/03/09032005.htm.

5 Cienfuegos EG, Orue MA, Briones M, Martínez JC. Estimación del comportamiento productivo y parámetros genéticos de características predestete en bovinos de carne (Bos taurus) y sus cruzas, VIII Región, Chile. Arch Med Vet 2006; 38(1): 69-75.

6 Quintero JC, Triana JG, Quijano JH, Arboleda EM. Influencia de la inclusión del efecto materno en la estimación de 
parámetros genéticos del peso al destete en un hato de ganado de carne. Rev Col Cienc Pec 2007; 20: 117-123.

7 Dodenhoff J, Van Vleck LD, Gregory KE. Estimation of Direct, Maternal, and Grandmaternal Genetic Effects for Weaning Weight in Several Breeds of Beef Cattle. J Anim Sci 1999; 77: 840-845.

8 Boldman KA, Kriese LD, Van Vleck LP, Kachman SD. A manual for use of MTDFREML. A set of programs to obtain estimates of variance and covariances. ARS, USDA , Washington, D.C. 1995

9 Botero FM. Ganado Blanco Orejinegro. En. Razas Criollas Colombianas. Instituto Colombiano Agropecuario (Ed.) Bogotá, Colombia. Manual de Asistencia Técnica 1976; 21: 17-61.

10 Ossa G., Pérez J. Efecto del medio y de la herencia sobre los pesos al nacer, destete y 16 meses de edad en la raza costeño con cuernos. Rev.MVZ Córdoba 2002; 7(1): 143-147.

11 Ospina A. Características del ganado Blanco Orejinegro. Agric Trop 1950; 6(2): 12-19.

12 Hernández BG. Las razas criollas colombianas para la producción de carne. Recursos Genéticos animales en América Latina. Ganado Criollo y especies de altura. Roma, Italia, FAO, 1981; 22: 5276. [Fecha de acceso 30 de agosto del 2007]. URL disponible en: http:// www.fao.org/docrep/009/ah223s/ AH223S07.htm\#ch4.6

13 Plasse D. El uso del ganado criollo en programas de cruzamiento para la producción de carne en América Latina. Recursos Genéticos animales en América Latina. Ganado criollo y especies de altura. Roma, Italia, FAO. 1981; 22: 77. [Fecha de acceso 30 de agosto del 2007]. URL disponible en: http:// www.fao.org/docrep/009/ah223s/ AH223S08.htm\#ch4. 7

14 Martínez G. El ganado criollo colombiano Blanco Orejinegro (BON). Animal Genetic
Resources Information. FAO and UNEP, Roma, Italia 1992; 9: 27-36. [Fecha de acceso 30 de agosto del 2007]. URL disponible en: http: //lprdad.fao.org/cgibin/getblob.cgi? sid = 85b9b26130 610878fd46be05cfaf90b8,50005995

15 Ossa G, Suarez M, Pérez J. Efectos del medio y la herencia sobre el peso destete de terneros de la raza Romosinuano. Rev.MVZ Córdoba 2005; 10(2): 673-683.

16 Martínez-González J , Castillo-Rodríguez $S$, Lucero-Magaña F, Ortega-Rivas E. Influencias ambientales y heredabilidad para características de crecimiento en ganado Sardo Negro en México. Rev Zoot Trop 2007; 25(1): 1-7.

17 Martínez G. El ganado criollo Romosinuano (Romo). Animal Genetic Resources Information. FAO and UNEP 1998; 24: 111. (Fecha de acceso 30 de agosto del 2007). Disponible en: http:// agtr.ilri.cgiar.org/Library/docs/ agri24_98.pdf.

18 Martínez G, Frahman RR, Buchanan DS, Geisert RD. Caracterización de la raza criolla Blanco Orejinegro (BON). I. Comportamiento reproductivo y parámetros genéticos del crecimiento predestete. Rev ICA (Col) 1989; 24(4): 270-282.

19 Kaps M, Herring W, Lamberson E. Genetic and environmental parameters for mature weight in Angus cattle. J Anim Sci 1999; 77: 569-574.

20 Martins G, Martins R, Melo F, Lima Braga $R$. Influência de fatores genéticos e de meio sobre o crescimento de bovinos da aça Nelore no estado do Maranhão. Rev. Bras. Zootec. 2000 29(1) [Fecha de acceso 31 de agosto del 2007]. URL disponible en: http://www.scielo.br/ scielo. php?script=sci_arttext\&pid=S1516$3598200000010001 \overline{4}$.

21 McManus C, Saueressig M, Falcão R, Serrano G, Marcelino K, Paludo G. Componentes reprodutivos e produtivos no rebanho de corte da Embrapa cerrados. Rev Bras Zootec 2002; 31(2): 648-657. 
22 Splan RK, Cundiff LV, Dikeman ME, Van Vleck LD. Estimates of parameters between direct and maternal genetic effects for weaning weight and direct genetic effects for carcass traits in crossbred cattle. J Anim Sci 2002; 80: 3107-3111.

23 Elzo MA, Wakeman DL. Covariance components and prediction for additive and nonadditive preweaning growth genetic effects in an angus-brahman multibreed herd. J Anim Sci 1998; 76: 1290-1302.

24 De Souza JC, Gadini CH, Da Silva OC, Ramos AA, Euclides Filho K, De Alencar MM, Ferraz Filho PB, Van Vleck LD. Estimates of genetic parameters and evaluation of genotype $x$ environment interaction for weaning weight in Nellore cattle. Arch Latinoam Prod Anim 2003; 11(2): 94-100.

25 Elzo MA, Martínez G, Gonzáles F, Huertas $\mathrm{H}$. Variabilidad y predicciones genéticas aditivas, no aditivas y totales para la producción de ganado de carne en el rebaño multirracial Sanmartinero-Cebú de la Libertad. Revista Corpoica 2001 julio; 3(2): 51-64.
26 Reyes AB, Lobo RB, Oliveira HN. Estimativas de (co)varianzas y DEPs por el modelo animal bicaracter para los pesos y perímetro escrotal de ganado Nelore en Brasil. XIV Reunión ALPA, Buenos Aires, Argentina. Memorias; 1995; 926-930.

27 Meyer K. Estimates of genetic parameters for weaning weight of beef cattle accounting for direct-maternal environmental covariances. University of New England, Australia. 2001. (Fecha de acceso 30 de agosto del 2007); Disponible en: http://agbu.une.edu.au/ $\sim$ kmeyer/PublishedPaps/FalcMod.pdf.

28 Nuñez-Dominguez R, Van Vleck, LD, Cundiff LV. Breed comparisons for growth traits adjusted for within-breed genetic trend using expected progeny differences. J Anim Sci 1993; 71: 14191428.

29 Barkhouse KL, Van Vleck LD, Cundiff LV, Buchanan DS, Marshall DM. Comparison of sire breed solutions for growth traits adjusted by mean expected progeny differences to a 1993 base. J Anim Sci 1998; 76: 22872293. 